\title{
Chez les Matacos du Chaco argentin. Hommes et femmes dans un processus de colonisation tardive
}

The Matacos in the Chaco (Argentina). Men and women in a late colonial context

Ana A. Teruel

\section{(2) OpenEdition}

12 Journals

Édition électronique

URL : https://journals.openedition.org/clio/10070

DOI : $10.4000 /$ clio. 10070

ISSN : 1777-5299

Éditeur

Belin

Édition imprimée

Date de publication : 1 mai 2011

Pagination : 193-209

ISBN : 978-2-8107-0157-5

ISSN : $1252-7017$

Référence électronique

Ana A. Teruel, «Chez les Matacos du Chaco argentin. Hommes et femmes dans un processus de colonisation tardive », Clio. Femmes, Genre, Histoire [En ligne], 33 | 2011, mis en ligne le 01 mai 2013, consulté le 01 mai 2022. URL : http://journals.openedition.org/clio/10070 ; DOI : https://doi.org/ 10.4000/clio. 10070 


\section{Documents}

\section{Chez les Matacos du Chaco argentin. Hommes et femmes dans un processus de colonisation tardive*}

Ana A. TERUEL

Le Chaco, une vaste pénéplaine située entre la cordillère andine et le bouclier brésilien, est partagé aujourd'hui entre la Bolivie, le Paraguay, l'Argentine, et pour une moindre part le Brésil. Il compte parmi les nombreux territoires ayant fonctionné comme "frontière » dans l'histoire latino-américaine, tant à l'époque des empires coloniaux du $\mathrm{XVI}^{\mathrm{e}}$ au XVIII ${ }^{\mathrm{e}}$ siècle qu'à la suite des indépendances républicaines au début du XIX ${ }^{\mathrm{e}}$ siècle. Pour ce qui est de la région absorbée par l'Argentine, sa conquête effective s'est achevée tardivement, au début du XX $\mathrm{X}^{\mathrm{e}}$ siècle.

$\mathrm{La}$ «frontière » telle que nous l'entendons ici n'est pas une limite interétatique (border en anglais), mais une ligne d'expansion « interne » d'un État (en anglais: frontier) dans un espace habité par des aborigènes autonomes, et sur lequel l'État n'exerçait pas de contrôle réel. Perçues et désignées par les pouvoirs publics argentins au XIX siècle comme des "frontières internes ", l'expression contenait aussi la notion d'un "autre» inclus et simultanément étranger, différent - un «autre» stigmatisé comme barbare, comme sauvage ou bien, alternativement, nié.

* Traduit de l'espagnol (argentin) par Luc Capdevila. 
Dans ces circonstances, étudier les relations de genre dans des sociétés autochtones et leurs modifications produites par la colonisation impulsée par l'État national est une tâche complexe. En premier lieu cela implique de rompre avec les catégories coloniales homogénéisatrices, en particulier celle de l'«Indien », pour travailler avec les différents groupes ethniques et distinguer leurs particularités, prendre en compte la diversité de leurs relations sociales, et des identités de genre, sans perdre de vue les conditions du contact caractéristiques de la situation de frontière consistant dans des négociations, des échanges et des rapports de soumission. Cette analyse implique de se détacher de la représentation de la frontière réduite à un front belliqueux composé d'acteurs essentiellement masculins, afin d'observer la multiplicité des relations qui se sont développées dans cet espace; même dans les situations de guerre il faut débusquer les femmes au sein des populations natives qui souvent se comptaient par « lances » (lanzas), c'est-à-dire par guerriers mâles.

Aussi nous proposons de nous situer dans une portion de la frontière chaquéenne sous juridiction argentine, à des moments immédiatement antérieurs et postérieurs aux campagnes militaires engagées entre 1884 et 1911 pour soumettre les populations indigènes. Nous travaillons ainsi dans une région de la frontière en essayant de retrouver le prisme à travers lequel des acteurs du processus de colonisation ont observé les hommes et les femmes aborigènes.

Il est inutile de préciser que les études historiques sur les relations de genre à la frontière chaquéenne n'abondent pas. Les sources sont rares sur ce thème. Les femmes apparaissent en général en toile de fond des descriptions des villages indigènes et des tableaux de la vie quotidienne. Les témoignages les plus détaillés émanent des ethnologues, des voyageurs et d'autres scientifiques de l'époque qui, souvent, décrivent précisément les relations entre les garçons et les filles, entre les adultes et les enfants. Un autre type de sources est intéressant car il émane de la volonté d'agir pour «civiliser» et «discipliner» les indigènes, en imposant de nouvelles règles culturelles et des comportements censés être souhaitables pour les garçons et pour les filles. C'est le cas des deux textes que nous avons 
retenus. Ils proviennent de représentants des institutions qui assumèrent cette mission, de manière plus ou moins importante, dans ces territoires.

Dans un premier temps, il s'agit des missions religieuses. Elles jouèrent un rôle important pour étendre et consolider la frontière avec la volonté d'incorporer les indigènes à la société dominante. En effet, malgré la croissance du laïcisme républicain au sein de la classe dirigeante dans la seconde moitié du XIXe siècle, l'État argentin continua de leur confier cette fonction. À ce titre, nous présentons le regard d'un père franciscain des missions implantées sur les rives du fleuve Bermejo pour évangéliser les Matacos, à la bordure occidentale du Chaco ${ }^{1}$.

Dans un deuxième temps, nous nous intéresserons à un autre regard : celui d'un fonctionnaire de l'État national chargé, au début du $\mathrm{XX}^{\mathrm{e}}$ siècle, de faire respecter les lois protectrices récentes des travailleurs indigènes des établissements productifs installés près de la frontière. Au cours de l'avancée du front de colonisation, les Matacos, à l'identique des autres groupes chaquéens, ont été recrutés massivement comme brassiers par les raffineries sucrières. Les dirigeants politiques y ont vu deux effets positifs : répondre à la rareté de la main-d'œuvre, et simultanément discipliner et incorporer les indigènes à la société dominante. Mais les moyens pour parvenir à ces fins furent critiqués par certains fonctionnaires d'État, parmi lesquels l'Inspecteur du Département National du Travail, dont le témoignage sur les hommes et les femmes matacos est reproduit dans la deuxième partie de cette étude.

1 Mataco est un ethnonyme d'origine coloniale, le groupe concerné se disant aujourd'hui Wichí. Au XIXe siècle, les rives du fleuve Bermejo étaient principalement peuplées par des populations issues de la famille linguistique mataco-mataguayo. "Tierra adentro» (terme colonial désignant les territoires non conquis, dont la traduction littérale serait «l'intérieur des terres »), nettement plus éloignés des populations créoles que ne l'étaient les villages matacos, les foyers de peuplement les plus importants étaient ceux des Tobas et des Pilagás de la famille guaycurú. Dans les terres les plus fertiles de la frontière qui correspondaient aux bordures forestières des Yungas, les Chiriguanos, des agriculteurs d'origine tupíguaraní, étaient organisés dans des gros villages. 


\section{Dans les missions}

Sur les rives du Bermejo, à la bordure occidentale du Chaco argentin, six missions relevant du collège franciscain de San Diego de Salta furent fondées entre 1856 et 1900 afin de regrouper les Matacos qui avaient maintenu leurs campements près des foyers de colonisation. Colonia Rivadavia, l'établissement créole le plus important dans le voisinage des missions, était connu pour être un lieu majoritairement masculin. Le recensement réalisé seulement trois ans après sa fondation en $1865^{2}$, signale 433 habitants avec une proportion de 121 hommes pour 100 femmes, le taux de masculinité augmentant pour la classe des 30-49 ans. 21\% des foyers étaient composés de personnes sans lien familial entre elles, seulement $40 \%$ correspondaient à des familles nucléaires - une situation assez caractéristique de la frontière colonisée en grande partie par des migrants masculins. Cet environnement essentiellement masculin contrastait avec la situation à l'intérieur des missions. Fondée en 1859, celle de La Purísima Concepción del Bermejo réunissait, en 1871, 276 individus regroupés en 54 familles. Tous étaient enregistrés comme «Indien réduit ${ }^{3}$ de la race mataca ». Frère Remedi, chargé du dénombrement, précisait que :

Le patronyme, excepté pour quelques-uns qui en ont un, je l'ai remplacé avec le nom que chacun a dans sa langue et par lequel ils sont connus et identifiés. Pour quelques enfants qui n'en n'ont toujours pas, je leur ai attribué celui du père lorsqu'il s'agit de garçons, si ce sont des femmes celui de la mère. ${ }^{4}$ Tous ont un nom de chrétien, qui leur a été donné dans les missions, ou dans les établissements sucriers 5 .

La structure par sexes montre un indice de masculinité très bas de 68 hommes pour 100 femmes. Le déficit masculin est manifeste pour

2 Archives Historiques de Salta (AHS), dossier Gouvernement 1865, nº 4 . Recensement de 1865. Département de la Colonie Rivadavia/Partido del Pueblo.

3 Réduit dans le sens d'appartenant à une "réduction », c'est-à-dire un regroupement d'Indiens sédentarisés placés sous l'autorité des missionnaires (NdT).

4 Il s'agit d'une disposition issue du troisième concile de Lima établissant que lors du baptême le patronyme du père serait donné aux garçons, et celui de la mère aux filles cf. Saiz 1992.

5 Archives du couvent franciscain de Salta (ACFS), recensement de la population de La Purísima Concepción del Bermejo 1871. 
le groupe des 15-24 ans. Il s'agissait d'adultes jeunes qui partaient travailler régulièrement hors de la mission dans les estancias ${ }^{6}$ d'élevage et dans les raffineries sucrières des terres voisines de la province de Jujuy?

Dans ce contexte, il est nécessaire de signaler la rareté des références explicites concernant les femmes dans les documents émanant des frères missionnaires, dès lors qu'ils mentionnent les Matacas et les Matacos avec le terme générique de «les Indiens». La lecture des journaux des Franciscains donne le sentiment qu'ils n'enregistrent que les sujets brûlants qui, en général, impliquent seulement les hommes comme protagonistes, qu'ils soient créoles ou indigènes ${ }^{8}$. Les femmes sont mentionnées comme victimes de violences ou parce qu'elles ont transgressé une norme quelconque. Sur un total de trente cahiers écrits par les missionnaires, il est fait mention de rares situations impliquant des femmes. Nous apprenons ainsi qu'une Mataca, qui était sortie pour porter secours à son mari lors d'une bagarre avec un autre Mataco, reçut un coup de couteau à la pointe du poumon ${ }^{9}$; que furent condamnés au cep ${ }^{10}$ durant tout une journée les fils du cacique Ujnai qui s'étaient battus avec d'autres pour une femme, avec laquelle l'un d'entre eux voulait se marier sans son consentement; ou qu'une «china »11 fut châtiée parce qu'elle

6 Estancia: hacienda du Cône sud généralement spécialisée dans l'élevage extensif (NdT).

7 Dans les villages matacos restés en dehors de l'autorité des missions religieuses, les déplacements vers les raffineries de sucre, qui pouvaient durer de quatre à six mois, étaient massifs. Ils concernaient autant les hommes que les femmes accompagnées de leurs enfants. Dans les missions, par contre, seuls les hommes s'absentaient, et laissaient temporairement leurs femmes.

8 Les journaux inédits de la mission franciscaine du collège de San Diego sont analysés dans Teruel 2005.

9 ACFS, journal de La Purísima Concepción del Bermejo, 1871.

10 Le condamné au «cep » (cepo) était attaché à un poteau planté au centre de la mission et exposé pendant un temps donné sans que les passants puissent lui apporter le moindre réconfort $(\mathrm{Nd} \mathrm{T})$.

11 "China » était le terme utilisé par les créoles pour désigner les femmes indigènes, cf. Braunstein 2008. Fortement dépréciatif, il est employé encore aujourd'hui sous la forme du diminutif «chinita» (petite china) pour nommer les employées domestiques ou les femmes des milieux populaires. 
s'était enfuie de la mission avec un créole ${ }^{12}$. La fuite d'une femme pour s'unir à un homme était réprimandée avec sévérité ; lors de cet épisode, le missionnaire était allé jusqu'à la ville d'Orán pour la récupérer. Par ailleurs, il ne semble pas que les frères condamnèrent la pratique mataca qui faisait que la femme choisissait l'homme de manière systématique lorsqu'il s'agissait d'une union durable et non de circonstance ${ }^{13}$.

Comme dans de nombreuses situations de colonisation, la grande préoccupation des missionnaires était le contrôle de la sexualité des femmes et l'imposition de règles "morales » civilisatrices. Bien que la dénonciation du manque de pudeur concernât les femmes, l'accusation de luxure était réservée aux garçons qui se rendaient coupables d'inconstance dans les relations maritales durables.

Le peu de témoignages missionnaires réservant quelques passages étendus aux femmes éclaire davantage sur les représentations des missionnaires que sur les relations de genre. Dans ce texte publié comme étude ethnographique - à la différence d'autres écrits missionnaires dans lesquels prédomine le stéréotype du «bon sauvage »- Rafael Gobelli14 est très négatif envers les Matacos. Il affirme en particulier sa conviction que toute action civilisatrice en leur faveur est inutile. Il est également nécessaire de préciser pour une meilleure compréhension que lorsque ce texte fut écrit en 1914, les cinq autres missions avaient déjà succombé à leur déclin :

Un autre vice très répandu chez les Matacos, et qui, sans doute, influe puissamment pour dénerver leurs forces, les dégénérer et les envahir de maladies répugnantes, est la sensualité. Ils acquièrent ce vice dès l'enfance. Cela ne doit pas surprendre si l'on tient compte du fait que, dès le plus jeune âge, les enfants des deux sexes vaquent tout nus, et dorment dans le même état à côté de leurs parents et voisins, formant un tas répugnant de grands et de petits, d'hommes et de femmes, de célibataires et de mariés. Il est facile d'imaginer toutes les mauvaises

12 ACFS, journal de La Purísima Concepción del Bermejo, 1868.

13 Une pratique décrite pour les Chorotis par Nordenskïold 1912, et pour les Matacos actuels par Pérez Bugallo 1994.

14 Rafael Gobelli, né en 1862 à Alessandria (Italie), prit l'habit ecclésiastique en 1878 dans le couvent de Salta avant d'être ordonné prêtre en 1885. Il fut nommé Préfet de Misiones en 1910 et s'est occupé de l'administration de Nueva Pompeya. ACFS, carton Chaco «Stato personale del Collegio di Salta ». 
choses que voient et entendent les garçonnets et les fillettes dans cette école, et combien elles influencent pour susciter leurs vils instincts et leurs passions les plus dégradantes.

Pour cela, de manière très répandue, les jeunes, à peine venus à la puberté, ont déjà une femme, et portent imprimés sur leurs visages les signes de la dégradation.

Les pères et mères de famille ne se préoccupent absolument pas de l'éducation de leurs enfants: jamais ils ne les corrigent ni ne sont capables de les châtier, sinon que dès tout petits ils les laissent faire ce qu'ils veulent et aller selon leurs désirs. Le pire est que, lorsqu'ils ont des petites filles, eux-mêmes vont les offrir au premier venu. ${ }^{15}$

Comme je l'ai déjà consigné dans l'un de mes feuillets, les femmes matacas n'ont que très peu de considération pour la pudeur naturelle, qui est et devrait être la plus belle parure de leur sexe. Cependant il n'y a pas à s'étonner que cela se produise entre gens sauvages, lorsque l'on voit dans le monde civilisé tant d'avilissement, pareille corruption des coutumes, si peu de pudeur et de modestie dans la manière de se vêtir, de parler, et dans le travail.

Un homme bon observateur, qui passe quelques années chez les sauvages du Chaco, en revenant à la ville, en parcourant les grandes capitales, voyant tant de dénuement dans la manière de s'habiller, etc., etc., ne peut dire moins que : "La société moderne, oubliant les principes et les comportements de la morale chrétienne, est en train de revenir au paganisme et porte en triomphe toutes ses coutumes nauséabondes, bien que d'une manière plus ou moins voilées".

Aujourd'hui les ennemis de la religion travaillent avec acharnement pour rompre le lien indissoluble du mariage, aspirant à imposer le divorce absolu qui, sans nul doute, serait un véritable fléau pour la famille et pour la société. Si le divorce venait à être approuvé, la société par ce biais retournerait au paganisme et à la sauvagerie propre aux Indiens du Chaco. En effet, chez les Indiens sauvages existe le divorce absolu, plus simple et plus aisé que celui projeté chez nous autres, effectivement, il n'y est pas nécessaire de recourir au juge ni d'attendre sa sentence pour divorcer et se marier avec une autre. Un prétexte suffit ou une chose quelconque, même si elle est ridicule. J'ai connu des Indiens qui ont divorcé après avoir vécu unis durant de nombreuses années, parce que leur femme était laide; d'autres, parce qu'elle ne savait pas coudre, d'autres parce qu'elle ne savait pas laver les vêtements. Un jour je

15 [Note de Gobelli] «Je m'incline à croire que cela, ils l'ont appris de certaines personnes civilisées lorsqu'ils vont travailler dans les exploitations forestières (obrajes) et dans les raffineries sucrières (ingenios)». 
reprochais à un Indien de s'être uni à une autre femme, abandonnant la sienne à la dernière misère ainsi qu'un tout petit encore nourri au sein, il me répondit qu'il l'avait abandonnée car elle ne savait pas s'occuper de ses enfants!!! ... Comme si entre ses mains il y avait le pouvoir d'empêcher que la maladie leur prenne la vie! Combien de ces cas verrons-nous, si par malheur est approuvé le divorce!

Il n'est pas rare qu'un Indien divorce ainsi, et aille s'unir à la première après avoir abandonné la deuxième ou la troisième avec sa propre progéniture, les laissant dans la plus grande misère et à l'abandon ${ }^{16}$.

Au-delà de l'entreprise de moralisation, les frères insistent sur la nécessité de «dresser» les Matacos et d'en faire des travailleurs disciplinés, comme partie intégrante de la mission civilisatrice. Les rôles sexués sont ainsi clairement définis : "Dès leur incorporation à la mission, les hommes seront dédiés au travail, les femmes s'occuperont de leurs tâches domestiques et les enfants assisteront à l'école $»^{17}$. Tous les espoirs étaient placés dans les enfants, garçons et filles, que, dans la mesure du possible, on essayait de séparer de leurs parents pour leur assurer «une bonne éducation». Ainsi, à Concepción del Bermejo, "Micaela Nieto de Caraparí, commença à enseigner aux chinitas à prier et à coudre $»^{18}$; et à Nueva Pompeya le déjà mentionné Frère Gobelli affirmait que :

J'ai placé deux petits Indiens au collège de Artes y Oficios des Salésiens à Salta, et deux fillettes matacas au collège de Santa Rosa de la même ville. J'espère que ces garçons et filles lorsqu'ils reviendront à la mission, lui seront utiles et serviront de modèle aux autres ${ }^{19}$.

Le règlement de la mission distingue clairement entre « les tâches domestiques» et «le travail». Cette dernière notion excluait les activités réalisées dans la brousse (monte): la chasse et la pêche

16 L'«Estudio etnográfico sobre los indios matacos » constitue la troisième partie de Memorias de mi Prefectura y apuntes sobre el Chaco, de Rafael Gobelli, publié à Salta en 1914. Nous citons ici la réédition dans Teruel 1995 : 133-134.

17 Règlement pour la mission de San Francisco de Laihí (1914) étendu par décision du ministère de l'Intérieur (11 janvier 1915) à celle de Nueva Pompeya. Bulletin de la Dirección General de Territorios Nacionales, septembre 1914, t. 1. p. 22.

18 ACFS, journal de La Purísima Concepción del Bermejo, 1870.

19 Gobelli 1914 in Teruel $1995: 136$. 
(masculines) ou la collecte (de manière prédominante féminine) ${ }^{20}$. Revenons au témoignage de Gobelli :

Comme je l'ai déjà dit, l'Indien mataco, oisif et de caractère fainéant, ne se préoccupe pas de se procurer, par le travail, les ressources nécessaires à sa subsistance et à celle de sa famille ; mais, au milieu de sa pauvreté, il se sent dans son élément. Certains diront que le Mataco considère le travail comme une déchéance. [...]

Malheureusement, les bois avec leur variété de fruits sylvestres, l'abondance des oiseaux et des poissons dans les lagunes et les fleuves Teuco et Bermejo, contribuent à maintenir ces malheureux Matacos dans un état de désœuvrement et de barbarie, les incitant à l'oisiveté et à l'horreur du travail. [...].

Celles qui cherchent des racines et des fruits dans les bois sont les femmes. Ce sont elles aussi qui transportent l'eau et le bois, celles qui cuisinent, etc. Au cours des déplacements, les Indiennes sont comme des bêtes de somme, en effet, elles portent le mortier, la marmite et toutes leurs hardes. Elles ont un système spécial pour porter leurs petits. Elles les calent avec les jambes sur leur flanc et les soutiennent avec le bras. Si elles ont deux ou trois petits, au cours des déplacements elles en prennent un selon la manière déjà indiquée, un autre est porté sur le dos et le troisième elles le mettent dans une llica ou un sac en chaguar ${ }^{21}$. Pendant que la pauvre femme est chargée comme un âne, l'Indien marche reposé, tenant seulement une escopette, ou sur ses terres, l'arc et les flèches. [...]

En effet, les hommes matacos qui errent de part les déserts ${ }^{22}$ du Chaco ne savent rien faire, si on excepte les flèches, les filets et les lances qu'ils utilisent pour se battre, chasser et pêcher. En revanche, les femmes sont un peu plus habiles et ingénieuses, en effet elles savent filer, tisser, fabriquer des poteries, etc., bien que selon des méthodes en rapport avec

20 Sur les activités économiques des Matacos on peut se reporter à Metraux 1944, Mashnshnek 1974. On trouvera une description détaillée de la spécialisation selon le sexe chez les Chorotis dans Nordenskiold 1912.

21 Type d'agave (NdT).

22 Désert ne correspond pas dans le cas présent à un milieu géographique. Il s'agit d'une catégorie coloniale qui remonte à la notion paléochrétienne de désert spirituel. Dans le Cône sud au XIXe et encore au début du XXe siècle l'emploi du substantif désert évoque les terres indiennes restées en marge du processus de conquête $(\mathrm{NdT})$. 
leur ignorance et leur stupidité. Avec la fibre du caraguatá ${ }^{23}$ elles fabriquent des attaches et des ficelles assez résistantes. Pour élaborer la ficelle et la corde, en raison du manque d'instruments nécessaires, elles se servent des mains et de leurs jambes pelées, sur lesquelles elles roulent la fibre. Cette opération doit être assez douloureuse pour les malheureuses Indiennes. Avec la ficelle elles font des filets et des sacs dans lesquels elles recueillent les fruits des bois. Elles ont l'habitude de les teindre assez bien. En raison de l'absence d'aniline, elles utilisent l'écorce d'algarrobo ${ }^{24}$.

Dans notre mission de Nueva Pompeya, tous les Indiens savent bêcher et labourer la terre, semer, façonner des briques : certains savent couper et scier le bois. Nous avons aussi des Indiens menuisiers, un tailleur, un charron et forgeron, un tourneur, etc. $[\ldots] »^{25}$.

Le travail était lié aux activités économiques en relation avec la sédentarisation : l'agriculture (tâche partagée entre les hommes et les femmes chez les Matacos), l'exercice de métiers pour produire des biens et des services, et le travail comme brassier salarié pour lesquels on instruisait les garçons.

Il est intéressant d'observer comment ce modèle inculqué par les missionnaires reste actuel. Gordillo ${ }^{26}$, dans ses recherches avec une communauté du Chaco, observe dans les années 1990 que sous le concept de travail la majorité des Tobas considéraient seulement les activités salariées, l'horticulture, l'élevage et la production artisanale. La «marisca» (activité de collecte dans la brousse) et « le travail» sont des termes qui s'excluent mutuellement. Tandis que pour les hommes le désirable est le travail salarié (en particulier l'emploi public), pour les femmes l'artisanat est devenu un marqueur d'identité. Elles y trouvent l'une des rares activités qui leur permet de gagner de l'argent indépendamment de leurs maris. À la différence du travail masculin, nettement séparé de la nature, l'artisanat nécessite pour les femmes qu'elles circulent dans la brousse à la recherche de plantes de chaguar

23 Arbuste épineux dont les fibres végétales rustiques peuvent être tissées, et dont la pulpe fut également utilisée pour fabriquer du papier - d'ailleurs très résistant notamment par les Paraguayens dans les moments de grande pénurie (NdT).

24 Type de caroubier (NdT).

25 Gobelli 1914 in Teruel 1995 : 134-136.

26 Gordillo 2006. 
et de colorants naturels. Ainsi, l'artisanat associé à la brousse est devenu simultanément un marqueur de genre et d'ethnicité.

\section{Dans les raffineries sucrières}

Dès la fin du XVIII ${ }^{2}$ siècle les indigènes du Chaco regroupés dans les missions coloniales et les groupes «amis $»^{27}$ participaient à la récolte de la canne à sucre des haciendas de la frontière. Ils constituaient la main-d'œuvre la plus importante de la région. La demande de brassiers s'est accrue à partir des années 1870 lorsque, produit de la modernisation technologique, deux de ces haciendas, San Pedro et Ledesma, se transformèrent en entreprises agroindustrielles. Le besoin de brassiers provoqua un conflit avec les missions franciscaines dont il vient d'être question. Elles furent considérées par les entrepreneurs comme un obstacle à la liberté du recrutement d'indigènes. De ce fait, le pouvoir politique, lié aux intérêts sucriers et jugeant peu probants les résultats obtenus par les frères des missions, paria sur les raffineries de sucre comme institution civilisatrice ${ }^{28}$.

Le second témoignage éclaire cette étape. Il est issu de l'observation des Matacos et des Matacas dans leurs marches depuis les villages (tolderías) jusqu'aux raffineries où ils résidaient temporairement. Son auteur, José Elías Niklison, était inspecteur au Departamento Nacional del Trabajo ${ }^{29}$. Il était chargé de surveiller les

27 Les «Indiens amis »: ainsi étaient désignés par les autorités espagnoles, puis républicaines, les groupes amérindiens avec lesquels des alliances avaient été négociées et avec lesquels des collaborations étaient nouées (NdT).

28 Teruel 2005.

29 Le Département National du Travail créé en 1907 avait pour juridiction la capitale fédérale et les «Territoires Nationaux». Comme son nom l'indiquait il ne pouvait intervenir dans les affaires des provinces. Les organismes spécifiques créés pour gérer les nouveaux territoires nationaux étaient : la Direction Générale des Territoires Nationaux (1912) chargée des relations avec les Indiens et de la superintendance des missions et des réductions, et la Commission Financière des Réductions d'Indiens, créée également en 1912 (à partir de 1916 dénommée Commission Honoraire des Réductions d'Indiens), dont les attributions se rapprochaient de celles des missions religieuses. Ces deux organismes dépendaient également du ministère de l'Intérieur. Le ministère de l'Intérieur avait reçu la responsabilité d'administrer à travers les «Territoires » dits 
conditions de travail des Matacos dans les entreprises sucrières. Son rapport date de 1917. Niklison est donc le contemporain de Gobelli. Néanmoins, sa perception des femmes matacas, auxquelles il réserve un chapitre entier, est à l'opposé de celle du frère, une des raisons pour lesquelles il nous semble intéressant de la reproduire ici :

Les "chinas", comme généralement on les nomme dans la région, sont d'un genre agréable et d'une robustesse et d'une activité qui forcent l'admiration de ceux qui connaissent leur vie sans repos dans les villages et dans les établissements agricoles et industriels où elles se rendent en compagnie de leurs hommes. Au travail, les administrateurs, les intendants et les contres-maitres (capataces) les considèrent très supérieures à ces mêmes Indiens [les hommes], ce qui n'est pas surprenant au regard des habitudes et des coutumes de la race qui imposent aux femmes, dès le plus jeune âge, l'obligation de stimuler la volonté et la vigueur de la force physique.

De tailles moyennes, plus basses que les hommes, elles se singularisent dans la jeunesse par l'harmonie de leurs formes et par la finesse de leur démarche, l'élégance toute naturelle chez certaines, qui rappelle celle des jeunes filles Chiriguanas et Tobas. Leurs mains, leurs pieds et leurs oreilles sont touts petits et bien structurés, lorsqu'ils ne sont pas prématurément déformés par la dureté de la vie dans le désert. Un détail qui s'impose toujours à l'attention des observateurs est la constitution de la magnifique ligne des bras au point de jonction avec les mains [...].

Le teint de leurs visages ronds, aux traits grossiers, présente les mêmes tons de bronze et de cuivre que les hommes. Les yeux sont noirs, mais plus grands, brillants, et expressifs que ceux des hommes, et les cheveux également abondants, épais et noirs. La taille des Matacas est fine et leurs hanches gracieusement arrondies. Sympathiques par le regard - un étrange mélange de timidité enfantine et de passion raffinée-, elles le sont plus encore avec leur sourire, qui invariablement laisse découvrir l'enchantement de leur bouche fraiche et la magnifique blancheur de leurs dents.

Tendres, affectueuses, passionnées, elles vivent intensément leurs fortes émotions et les durs travaux de leur race. Elles vieillissent, de ce fait, rapidement, comme les autres indigènes du Chaco et de Bolivie; et à l'âge mûr, et avec la vieillesse, elles ne conservent pas le plus petit vestige

« Nationaux » les terres colonisées à la fin du XIXe siècle sur les espaces indiens (Chaco, Patagonie, Terre de Feu). De ce fait ces territoires ne relevaient pas des institutions républicaines administrant les provinces de la fédération argentine. 
de ce que fut leur jeunesse. Les vieilles Matacas sont épouvantablement laides.

Travailleuses, immensément et étonnement travailleuses, tout le poids de la matérialité de l'existence est porté par ces simples et pauvres filles de la forêt. Elles construisent leurs maisons, les caractéristiques "buetes"; filent, teignent et tissent le chaguar, le coton et la laine; ravitaillent en eau et en bois leurs foyers; travaillent la terre et sèment; ramassent et entreposent l'algarroba, le mistol $^{30}$ et le vinal ${ }^{31}$; elles allaitent et surveillent leurs petits, elles pétrissent et mettent à cuire les splendides cruches et jarres (puruñar) aux lignes artistiques - un art dans lequel elles se révèlent bien supérieures aux Tobas -; elles préparent l'aloja - la boisson fermentée favorite de leurs pères, maris et frères -; dans les longues marches elles portent les enfants sur leurs épaules, l'équipement du campement et jusqu'aux chiens familiers ; et dans l'immense océan vert des plantations de Jujuy, elles accomplissent le même travail que les hommes, peut-être mieux qu'eux [...].

Elles se marient très jeunes et sont fidèles à leurs maris, comme quelqu'un l'a déjà dit, par affection, par éducation, par tradition ; et dans peu de cas peut-être par peur, car l'Indien est extrêmement jaloux et fier, et jamais il ne tolère la moindre inconduite de la part de sa femme. Mères, elles sont capables de sacrifier leur vie pour celle de leurs enfants, quel que soit le moment, et la tendresse qu'en tant que filles elles professent à leurs pères est proverbiale.

Elles exercent une influence positive sur le courage et la volonté des hommes. Elles ont, comme toutes les femmes qui savent aimer, telles les passionnées de toutes les races et de tous les temps, une intuition et une clairvoyance admirables. Les Indiens les consultent, les écoutent et leur obéissent, sûrs de la bonté et du suprême désintérêt de leurs indications et de leurs conseils.

Et ainsi, comme elles sont consultées, écoutées et obéies, elles sont aussi aimées, intensément aimées par les Indiens. Il est nécessaire de revenir sur l'opinion des Chrétiens, qui en les voyant chargées lors des marches épuisantes et contraintes aux dures et multiples tâches du campement, les perçoivent déprimées et abusées par les hommes.

La femme mataca n'est pas soumise, en réalité, à un traitement pire que celui de l'immense majorité des femmes dans notre société, et son rôle dans la communauté indigène est parfaitement adapté aux besoins sociaux et à son aptitude physique.

30 Type de jujubier (NdT).

31 Autre type de caroubier, parfois écrit « visnal », ou « algarrobo blanc » $(\mathrm{NdT})$. 
Les obligations auxquelles les Indiens doivent répondre leur imposent la liberté absolue et la facilité de mouvements. Eux, lors des marches, ne peuvent porter autre chose que leurs armes, car ils doivent chasser afin de s'approvisionner et de pourvoir les leurs en aliments, et parce qu'ils sont toujours dominés par la préoccupation naturelle du danger qui plane en permanence au-dessus de leurs têtes; le combat, la rencontre armée avec d'autres hommes. Autrement dit, comment pourraient-ils répondre à l'agression et se défendre si c'était eux qui étaient chargés ?

Par ailleurs, ce que les Matacas font dans les campements et dans les tribus, n'est rien d'autre que l'exacte reproduction de la vie active et industrieuse des paysannes européennes. "Dans tous les villages où je suis allé - écrivait un illustre explorateur étranger - j’ai été surpris dans l'ensemble des foyers paysans par la manière des femmes de s'occuper, en permanence elles sont employées à travailler."

On pourrait penser que le travail absorbant et épuisant auquel les "chinas" se consacrent, tuera ou neutralisera chez elles les attributs spirituels de leur sexe, pourtant, il n'en n'est rien, elles sont, avant tout et surtout, si on s'éloigne des idées reçues, profondément féminines. À leur manière et dans le cadre de l'ambiance particulière qui les entoure, elles se manifestent avec les sentiments, les goûts, et jusqu'à la coquetterie des femmes des autres races.

Dans les villages, leurs corps apparaissent presque toujours dans une lumineuse et chaste nudité. Ainsi elles se montrent aux regards, absolument sereines en raison de la coutume de la vie primitive des gens de la tribu ; mais suspectant la présence de personnes étrangères, elles se couvrent en toute hâte avec des gestes de pudeur équivalents à ceux des Chrétiennes. Dans la forêt, le vêtement des Matacas consiste dans une petite pièce de tissu qui enveloppe, serrée à la ceinture, le ventre et les cuisses. De la ceinture à la tête, tout juste si quelques fils et des petits disques d'os et de bois, faits par elles, interrompent à hauteur de la gorge et de la base des seins, l'obscure couleur de leurs bustes de bronze. Elles portent aussi, au moment de leur jeunesse, des bracelets de cuir grossiers, que, au moment du mariage, elles offrent au mari en gage d'alliance et de fidélité.

Dans les villes de la frontière et dans les zones de travail des entreprises sucrières du nord-ouest, elles portent de vulgaires jupes, des chemises et des blouses de tissus grossiers. Leur prédilection pour les couleurs vives, pour le rouge ardent en particulier, se vérifie par un seul détail: le foulard de coton ou de soie qu'elles nouent autour du cou ou avec lequel elles se couvrent la tête. 
Une grande diversité, non dépourvue d'harmonie et de goût, caractérise le travail des Indiennes. Les tissus de "chaguar" - les chemises sans manches, sorte de tunique, et les "yicas" 32 - sont beaux avec les dessins gracieux et colorés qui les décorent. Quant à ceux faits de laine et de coton - ponchos, manteaux, couvertures, jupes, sacs ou besaces - ils sont peut-être meilleurs que ceux des Tobas au regard de la régularité et du soin de la trame, mais inférieurs pour ce qui est des motifs et de la couleur $[\ldots]$.

Il est nécessaire de bien les connaitre, de se lier d'amitié avec les femmes matacas pour comprendre leur véritable caractère, sain, allègre et expansif dans de pareilles conditions. "Les 'chinas' dans des situations inhabituelles - quelqu'un a écrit - restent muettes et impassibles, mais entre elles, elles sont blagueuses et joueuses comme des enfants. Et cela est, en général, le propre des Indiens". Exact. Je l'ai vérifié avec le désir de bien connaitre l'âme et le cœur des populations que je devais étudier. Dans les moments de franche effusion et de tendre camaraderie, j'ai toujours vu les hommes et les femmes matacos allègres, aimables, généreux et bons comme des enfants; et les femmes, surtout, jamais n'oublient de répondre, spontanées et spirituelles, avec les expressions affectueuses qu'elles vous adressent dans leur langue ${ }^{33}$.

Le texte de l'inspecteur Niklison, bien qu'alourdi par les représentations de son époque et de son appartenance de genre, dénonçait en fait une situation : pour les Matacos et leurs femmes, il semait le doute sur la si fameuse œuvre de civilisation des raffineries de sucre ; une mission dont les entrepreneurs et leurs administrateurs se vantaient, en publiant quelques années plus tard, dans la presse locale, des affirmations comme celle-là :

Je crois que nous méritons une médaille d'or pour l'œuvre de civilisation que nous avons accomplie envers l'indiada ${ }^{34}$; pour ma part, je suis sûr que j'en mérite une douzaine pour les conversations, les contacts et les "parfums" que j'ai supportés durant toutes ces heures à m'occuper de ces gens ${ }^{35}$.

Le tableau rapide brossé pour cette étude permet d'entrevoir quelles furent les perceptions des relations de genre de la part de

\footnotetext{
32 Pièce de tissu en wichí (NdT).

33 Niklison 1989 [1917]: 57-60.

34 Indiada: la foule indienne ( $\mathrm{NdT})$.

35 El Heraldo, Jujuy, 12 avril 1921. Cité dans Lagos 1989, étude préliminaire à Niklison [1917].
} 
quelques acteurs du processus de colonisation des Matacos dans le nord-ouest argentin. Constatons finalement, à partir des différents témoignages, la variété et le volume des tâches qui incombaient aux Matacas, tant dans les campements que dans les missions et les raffineries. L'analyse des mutations des rôles et des relations nécessite une recherche plus approfondie, qui pourrait rendre compte également de la perception des hommes et des femmes selon leur appartenance de sexe. Ainsi, l'introduction des relations salariées où s'investirent majoritairement les garçons, mais qui a concerné également les femmes, est considérée aujourd'hui par les Chaquéennes comme un facteur positif qui leur a procuré plus d'autonomie à l'égard de leurs maris ${ }^{36}$. Or, ce fait a été aussi interprété comme un agent débilitant de l'identité aborigène. La question est complexe et problématique. Nous laissons pour le moment le débat ouvert.

\section{Bibliographie}

BRAUNSTEIN José, 2008, «La coutume du chineo en procès dans le Chaco argentin », in Carmen Bernand, Capucine Boidin \& Luc Capdevila (dir.), "Amériques métisses », CLIO. HFS, 27, Toulouse, PUM, p. 205-208.

GobelLi Rafael, 1914, «Estudio etnográfico sobre los indios matacos », in Memorias de mi Prefectura y Apuntes sobre el Chaco, Salta, Imprenta y Librería de Tula y Sanmillán. Réédition dans Ana Teruel (selec), 1995, Misioneros del Chaco occidental, Jujuy, CEIC.

Gordillo Gastón, 2006, En el Gran Chaco. Antropologias e Historias, Buenos Aires, Prometeo.

MashnshneK Celia O., 1974, «La economía de los Mataco del Chaco Argentino », Cuadernos Franciscanos, 35, Salta.

Metraux Alfred, 1944, «Estudios de etnografía chaquense. La vida económica de los indios del Chaco ", Anales del Instituto de Etnografia Americana, 5, Universidad Nacional de Cuyo.

36 Ainsi, Gordillo 2006 cite un entretien réalisé avec une femme toba qui se rappelait avec nostalgie le travail dans une raffinerie, car elle recevait directement la paye journalière sans en rendre compte à son mari. 
NiKLISON José Elías, 1989 [1917], «Investigación sobre los indios matacos trabajadores ", Boletin del Departamento Nacional de Trabajo, 35, Buenos Aires, Imprenta G. Pesce. Réédition Universidad Nacional de Jujuy, avec une étude préliminaire de Marcelo LAGos.

NordenSKÏOLD Erland, 1912, La vie des indiens dans le Chaco, Paris, L. Delagrave.

PÉrez Bugallo Rubén, 1994, "Instrumentos musicales del Chaco Argentino vinculados con la atracción sexual », Cuadernos del Instituto Nacional de Antropología y Pensamiento Latinoamericano, 15, Buenos Aires.

SAIz Félix, 1992, Los Colegios de Propaganda Fide en Hispanoamérica, Série V Centenario, Lima, Franciscanos Evangelizadores del Perú.

Teruel Ana, 2005, Misiones, economía y sociedad en la frontera chaqueña del Noroeste Argentino en el siglo XIX, Quilmes, Editorial de la Universidad Nacional de Quilmes. 